\title{
Intravenous lidocaine to prevent endothelial dysfunction after major abdominal surgery: a randomized controlled pilot trial
}

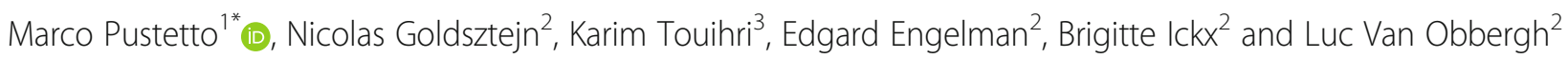

\begin{abstract}
Background: Major abdominal surgery is associated with endothelial glycocalyx disruption. The anti-inflammatory effects of lidocaine were recently associated with endothelial barrier protection.

Methods: This was a single-centre, parallel group, randomized, controlled, double blind, pilot trial. Forty adult patients scheduled for major abdominal surgery were included between December 2016 and March 2017 in the setting of a University Hospital in Brussels (Belgium); reasons for non-inclusion were planned liver resection and conditions associated to increased risk of local anesthetics systemic toxicity. Patients were randomized to receive either lidocaine by continuous intravenous administration or an equivalent volume of $0.9 \%$ saline.

The primary endpoint was the postoperative syndecan-1 concentration (difference between groups). Near-infrared spectroscopy of the thenar eminence in association with the vascular occlusion test, and contemporary analysis of flow-mediated dilation of the brachial artery were the secondary outcomes, along with haemodynamic data. Blood samples and data were collected before surgery (T0), and at 1-3 h (T1) and $24 \mathrm{~h}$ (T2) post-surgery.
\end{abstract}

Results: Syndecan-1 concentration increased significantly post-surgery $(P<0.001)$, but without any difference between groups. The near-infrared spectroscopy-derived and flow-mediated dilation-derived variables showed minor changes unrelated to group assignment. Compared with the placebo group, the intervention group had a significantly lower peri-operative mean arterial pressure and cardiac index, despite equally conducted goal-directed haemodynamic management. Postoperative lactate concentrations were similar between groups.

Conclusions: Lidocaine failed to have any effect on endothelial function. Since in comparisons to other types of clinical situations, syndecan-1 was only slightly upregulated, endothelial dysfunction after major abdominal surgery might be overestimated.

Trial registration: «ISRCTN Registry » identifier: ISRCTN63417725. Date: 15/06/2020. Retrospectively registered.

Keywords: Endothelial glycocalyx, Lidocaine, Major abdominal surgery, Endothelial dysfunction

\footnotetext{
* Correspondence: marco.pustetto@gmail.com

'Department of Anesthesiology, Centre Hospitalier Universitaire

Grenoble-Alpes, Boulevard de la Chantourne, 38700 Grenoble, France

Full list of author information is available at the end of the article
}

\section{$\triangle B M C$}

(c) The Author(s). 2020 Open Access This article is licensed under a Creative Commons Attribution 4.0 International License, which permits use, sharing, adaptation, distribution and reproduction in any medium or format, as long as you give appropriate credit to the original author(s) and the source, provide a link to the Creative Commons licence, and indicate if changes were made. The images or other third party material in this article are included in the article's Creative Commons licence, unless indicated otherwise in a credit line to the material. If material is not included in the article's Creative Commons licence and your intended use is not permitted by statutory regulation or exceeds the permitted use, you will need to obtain permission directly from the copyright holder. To view a copy of this licence, visit http://creativecommons.org/licenses/by/4.0/ The Creative Commons Public Domain Dedication waiver (http://creativecommons.org/publicdomain/zero/1.0/) applies to the data made available in this article, unless otherwise stated in a credit line to the data. 


\section{Background}

Amide-linked local anesthetics (LAs) such as lidocaine and ropivacaine have an anti-inflammatory effect [1-5]. Their use in the peri-operative setting for this purpose was proposed more than one decade ago [1-5]. Continuous intravenous infusion of lidocaine reduced pain scores after major abdominal surgery, improves gastrointestinal recovery and potentially reduces the in-hospital length of stay [6]. Whether these effects are related to sodium-channel blockade is debated. However, the inhibition of tumor necrosis factor alpha (TNF- $\alpha$ ) signaling pathway by LAs was recently associated with endothelial barrier protection in vitro [5].

The endothelial glycocalyx (EG) is a key component of the endothelial surface layer. It is essential for the regulation of the vascular barrier function, interaction between endothelial cells and blood cells, and in the transmission of shear stress. Extensive tissue trauma, hypervolemia, systemic and regional ischemia-reperfusion injury and shock from any origin have been associated with EG disruption. Systemic inflammation appears to have a causative role in EG shedding, with the exception of acute hypervolemia, for which the primary insult may be a direct mechanical injury $[7,8]$. However, a direct cause-effect relationship between inflammation and EG shedding is difficult to establish. Damage of the EG leads to tissue edema (i.e. decreased tissue access to oxygen and nutrients), increased interaction with leukocytes and platelets, and increased inflammation in a vicious cycle [7].

The same clinical scenarios are often associated with notable microcirculatory alterations. In these settings, markers of EG disruption and microvascular derangement are correlated with patients' morbidity and mortality [911]. Major abdominal surgery represents a scheduled but severe tissue trauma, is associated with important fluid shifts and an inflammatory state and actually induces EG shedding and disturbs endothelial function $[12,13]$.

Little information exists regarding possible restoration of the EG. To date, the most effective therapeutic strategy may be to preserve endothelial function and the EG; many different interventions have been proposed [10, 14-17]. Some of these are reasonable fluid administration [14], use of antiinflammatory drugs [15], and possibly goal-directed haemodynamic management [18]. The results achieved with hydrocortisone [15] support the idea that inflammation could be at least one mechanism that disrupts the EG. Antiinflammatory drugs may therefore be used for this specific purpose. We hereby hypothesise that intravenous continuous infusion of lidocaine can protect the EG and preserve endothelial function during major abdominal surgery.

\section{Methods}

\section{Trial design}

This was a randomized controlled pilot trial that compared two parallel groups for superiority of intervention versus placebo. Ethical approval for this study (Ethics Committee No. P2016/404/2016-003918-27) was provided by the Ethics Committee Erasme Hospital, 808 route de Lennik, B-1070 Brussels, Belgium (Chairperson Prof J.-M. Boeynaems) on 24 October 2016. The study was retrospectively registered in the ISRCTN Registry (Identifier: ISRCTN63417725) the 15/06/2020. All included patients signed a written informed consent before participation. This study adheres to CONSORT guidelines for reporting clinical trials (see Additional file 4: « CONSORT Checklist »).

\section{Participants}

Adult ( $>18$ years old) patients scheduled for elective major abdominal surgery were investigated for eligibility. Patients scheduled for colonic or bariatric surgery were considered to have a 'moderate risk', and thus not included in the eligibility screening. Patients scheduled for hepatic resection were excluded to prevent potential accumulation of lidocaine because of unpredictable changes in its pharmacokinetics. Patients who were to be managed with combined epidural and general anesthesia (e.g. esophagectomy) were excluded because of potential parallel administration of another LA. Patients presenting one or more of the following medical conditions were excluded because of increased risk of lidocaine accumulation and/or local anesthetics systemic toxicity: severe heart conduction blocks without implantable pacemaker, severe liver and kidney insufficiency (Kidney Disease: Improving Global Outcomes [KDIGO] stage $>3 \mathrm{a}$ ), acute heart failure, and known allergic reactions to any amide-linked LAs. Patients with atrial fibrillation were also excluded because it was impossible to follow the fluid administration protocol (discussed in the 'Interventions' section).

\section{Study setting}

This study was conducted at Erasme Hospital (Brussels, Belgium), a tertiary healthcare institution of the Universite Libre de Bruxelles. Patients were enrolled from December 2016 to March 2017.

\section{Interventions}

Patients allocated to the intervention (LIDO) group received $1.5 \mathrm{mg} \mathrm{kg}^{-1}$ (total body weight [TBW]) of $1 \%$ lidocaine (Xylocaïne; AstraZeneca, Cambridge, United Kingdom) just before anesthesia induction, which was immediately followed by a $2 \mathrm{mg} \mathrm{kg}^{-1} \mathrm{~h}^{-1}$ (TBW) continuous intravenous infusion until skin closure. Patients allocated to the placebo (PLA) group received an equivalent volume of $0.9 \%$ saline $\left(0.15 \mathrm{ml} \mathrm{kg}^{-1}\right.$ bolus and $0.2 \mathrm{ml} \mathrm{kg}^{-1} \mathrm{~h}^{-1}$ continuous intravenous infusion). Anesthesia protocol was standardized for both groups. When indicated, an intrathecal injection of $0.1-0.3 \mathrm{mg}$ of morphine was 
administered before induction. The latter was achieved using propofol, remifentanil (with target-controlled infusion $[\mathrm{TCI}]$ ) and a neuromuscular blocking agent (usually rocuronium or cisatracurium). Every patient received dexamethasone $(10 \mathrm{mg})$. Maintenance was achieved using desflurane and remifentanil (TCI), guided to maintain the Bispectral Index (BIS ${ }^{\mathrm{m}}$; Aspect Medical Systems, Norwood, MA, USA) readings between 40 and 60 with $0 \%$ of burst suppression rate. Hemodynamics were managed with a goal-directed therapy (GDT) protocol, based on stroke volume variation (SVV, measured using the Flo$\mathrm{Trac}^{\mathrm{nt}}$ system [EV1000; Edwards Lifesciences Corp., Irvine, CA, USA]) and mean arterial pressure (MAP). Plasmalyte (Baxter, Deerfield, IL) administered at $2 \mathrm{ml} \mathrm{kg}^{-1} \mathrm{~h}^{-1}$ was the basal infusion. Triggers for fluid bolus administration ( $250 \mathrm{ml}$ of crystalloids in $10 \mathrm{~min}$ ) and for catecholamine optimization were $\mathrm{SVV} \geq 13 \%$ and $\mathrm{MAP}<70 \mathrm{mmHg}$ (Fig. 1), respectively. When to transfuse hemoderivates and administer colloid solutions and the management of postoperative analgesia were at the discretion of the anesthetist in charge of the patient.

\section{Data collection}

Endothelial function and the EG were investigated using three techniques: concentration of syndecan-1, measurement of the tissue oxygen saturation $\left(\mathrm{StO}_{2}\right)$ during the vascular occlusion test (VOT), and contemporary measurement of flow-mediated dilation (FMD). Data and blood samples were collected before surgery (T0), at 1-
$3 \mathrm{~h}$ post-surgery in the recovery room (T1) and $24 \mathrm{~h}$ post-surgery in the surgical ward (T2).

The concentration of syndecan-1, a marker of EG shedding, was measured by classic sandwich enzymelinked immunosorbent assay (ELISA), based on the manufacturer's instructions (Syndecan-1 ELISA kit; Tebubio, Boechout, Belgium). Blood samples were collected in dry tubes, centrifuged to obtain the serum and stored at $-80^{\circ} \mathrm{C}$ for a maximum of 6 months before final analysis.

The $\mathrm{StO}_{2}$ was measured continuously (every $2 \mathrm{~s}$ ) and noninvasively using near-infrared spectroscopy (NIRS) (ForeSight ${ }^{\circ}$; CASMED ${ }^{\circ}$; CAS Medical Systems, Inc., Branford, CT, USA) on the thenar eminence, as previously described [11]. At the same time, the diameter of the brachial artery and flow velocity were measured continuously by using a $5-12 \mathrm{MHz}$ linear ultrasonography transducer (Sparq ${ }^{\circ}$; Phillips, Amsterdam, the Netherlands), which was applied to the upper arm with mechanical support for image stabilisation (Image 1 in «Additional File 1 » shows the standard set up for a participant). Baseline values were acquired over $1 \mathrm{~min}$. A VOT was then conducted by rapidly inflating a pneumatic cuff, which was placed around the forearm, up to $200 \mathrm{mmHg}$ (or $50 \mathrm{mmHg}$ suprasystolic pressure). After $5 \mathrm{~min}$ the cuff was released, and the hyperemic response was evaluated for another $4 \mathrm{~min}$. The following $\mathrm{StO}_{2}$-derived variables were analysed: $\mathrm{StO}_{2}-$ baseline, $\mathrm{StO}_{2}$-ischemic slope and $\mathrm{StO}_{2}$-reperfusion slope (Image 2 in «Additional File 2 » shows the evolution of

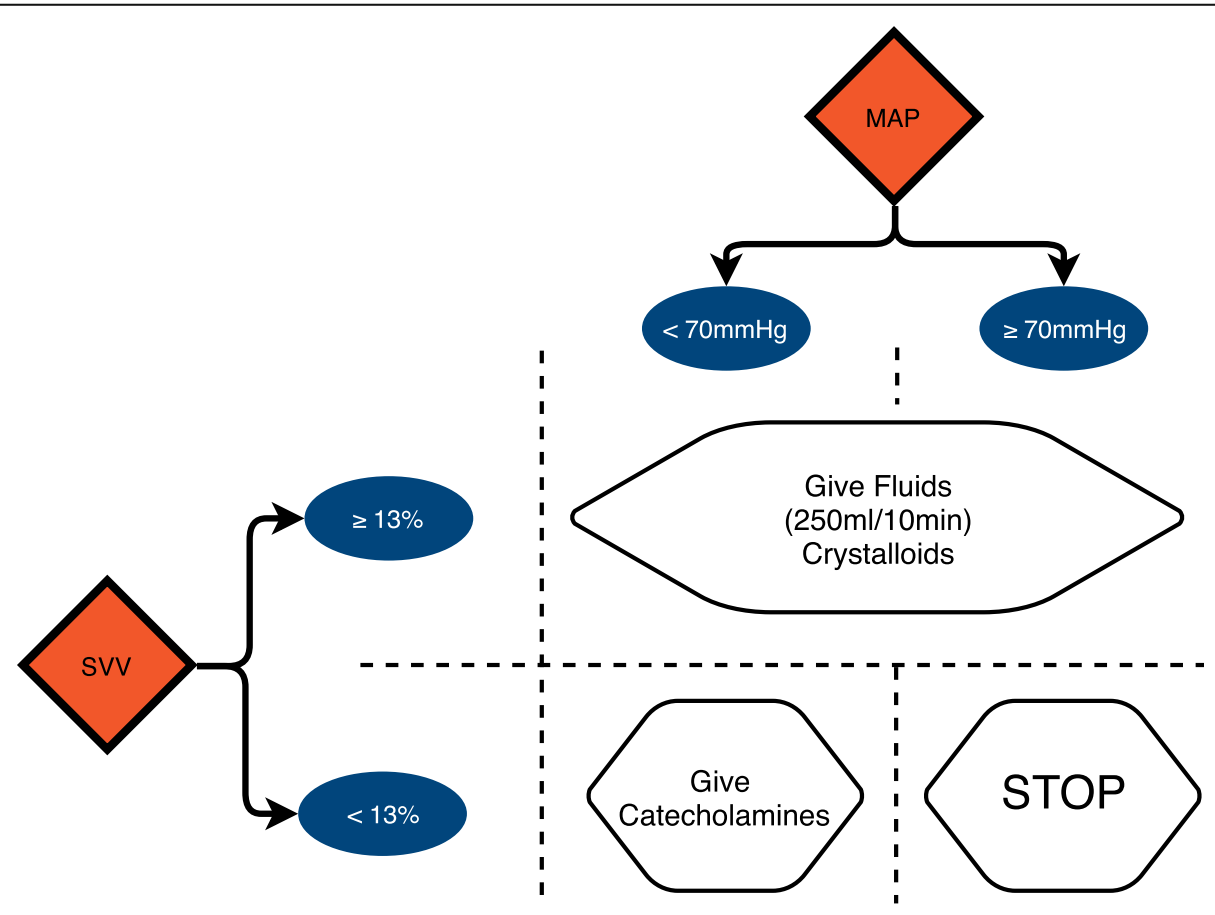

Fig. 1 Goal-directed haemodynamic protocol. Goal-directed protocol for the management of peri-operative fluids and haemodynamics: systolic volume variation (SW) and mean arterial pressure (MAP), as indicated on FloTrac ${ }^{\mathrm{TM}}$ (Edwards Lifesciences Corp., Irvine, CA, USA) 
$\mathrm{StO}_{2}$ during the test in one participant). The FMD was assessed by automated edge detection software (FMD Stu$\operatorname{dio}^{\mathrm{TM}}$ (CardioVascular Suite ${ }^{\mathrm{TM}}$ ); Quipu srl, Pisa, Italy) [19], based on the experts' guidelines [20].

The following FMD-derived variables were analysed: brachial artery baseline diameter, FMD-maximum (i.e. the maximal diameter during reperfusion) and the area under the curve of estimated shear rate of hyperaemic flow until FMD-max (Image 3 in " Additional File 3 » shows the evolution of the brachial artery diameter and shear rate during a test in one participant).

Data concerning fluid requirements were prospectively collected during surgery and during recovery in the postanesthesia care unit (PACU). Haemodynamic variables were collected only during surgery by the EV1000 clinical platform.

\section{Outcomes}

The primary endpoint was the evolution of the syndecan-1 concentration postoperatively in the LIDO group, compared with the PLA group (hereafter referred to as 'difference between groups'). Predefined secondary outcomes were the effect of lidocaine on NIRS- and FMD-derived variables (i.e. difference between groups); the influence of surgery on NIRS and FMD-derived variables and its association with group assignment (hereafter referred to as 'difference between times'); the correlation between glycocalyx, microcirculation and vascular reactivity at three time points; and the influence of group assignment on fluid requirements. Potential harmful effects of lidocaine were also systematically researched and reported. Haemodynamic variables, even if not originally included in secondary endpoints, were also taken in account for analysis and validation of compliance to the GDT protocol.

\section{Sample size}

We were unable to find any published study on the effects of lidocaine on endothelial function in the clinical setting, and evidence concerning alteration of EG in major abdominal surgery is scarce. Hence, we decided to perform a pilot study which included 40 patients with 20 patients for each group.

\section{Randomisation}

Participants were randomly assigned to one of two groups in a 1:1 ratio, based on Efron's biased coin randomisation procedure generated with NCSS v10 Statistical Software (2015, NCSS, Llc. Kaysville, UT, USA).

\section{Blinding}

Patients, healthcare providers, data collectors and outcome adjudicators were all blinded to group assignment. The physician in charge for generation of allocation sequence and concealment was not directly implicated in treatment administration or data collection.

\section{Statistical analysis}

Data are presented as the mean \pm the standard deviation. Data were compared between the groups using the Mann-Whitney test or by two-way analysis of variance (ANOVA) for repeated measures, as indicated. One factor was the study group and a second factor was time. For each variable, the three null hypotheses of the twoway ANOVA tests were that the means of the observations grouped by one factor would be the same; that the means of the observations grouped by the other factor would be the same; and that there would be no interaction between the two factors. The $P$ value would be indicated for the difference between groups (i.e., all time points together) or for the difference between times (i.e., all groups together), and for the interaction between groups and times. A Tukey-Kramer multi comparison test was performed to examine all pairs of treatment means. The other continuous non longitudinal variables were compared with the Mann-Whitney test. For all tests, $P<0.05$ was statistically significant. These computations were performed using the software package Systat version 5.0 for DOS (Systat, Inc., Evanston, IL, USA).

\section{Results}

We assessed 68 patients for eligibility and excluded 28 patients (seven patients had $\geq 1$ exclusion criteria, 13 patients refused to participate, and eight patients were excluded for technical problems related to the connection between the ultrasonography transducer and the FMD detection software and thus the impossibility to perform measurements on the brachial artery). Trial recruitment was stopped when 40 patients were included, 20 for each group, as planned. All patients were included in the final analysis (Fig. 2).

The patients' baseline characteristics for both groups are summarised in Table 1. Comorbidities and medications that influence baseline endothelial function and FMD measurements are reported in Table 1. The type of surgical intervention and whether it was conducted by laparotomy, laparoscopy or a combined procedure are reported in Table 2. Primary and secondary outcomes are presented in Table 3. Analysis of fluid requirements and hemodynamics are reported separately in Tables 4 and 5.

We failed to show any difference between groups for primary and secondary outcomes. Syndecan-1 increased modestly and equally between groups, and maximum levels were registered at T2 (i.e. $24 \mathrm{~h}$ post-surgery). Difference between times was statistically significant $(P<$ 


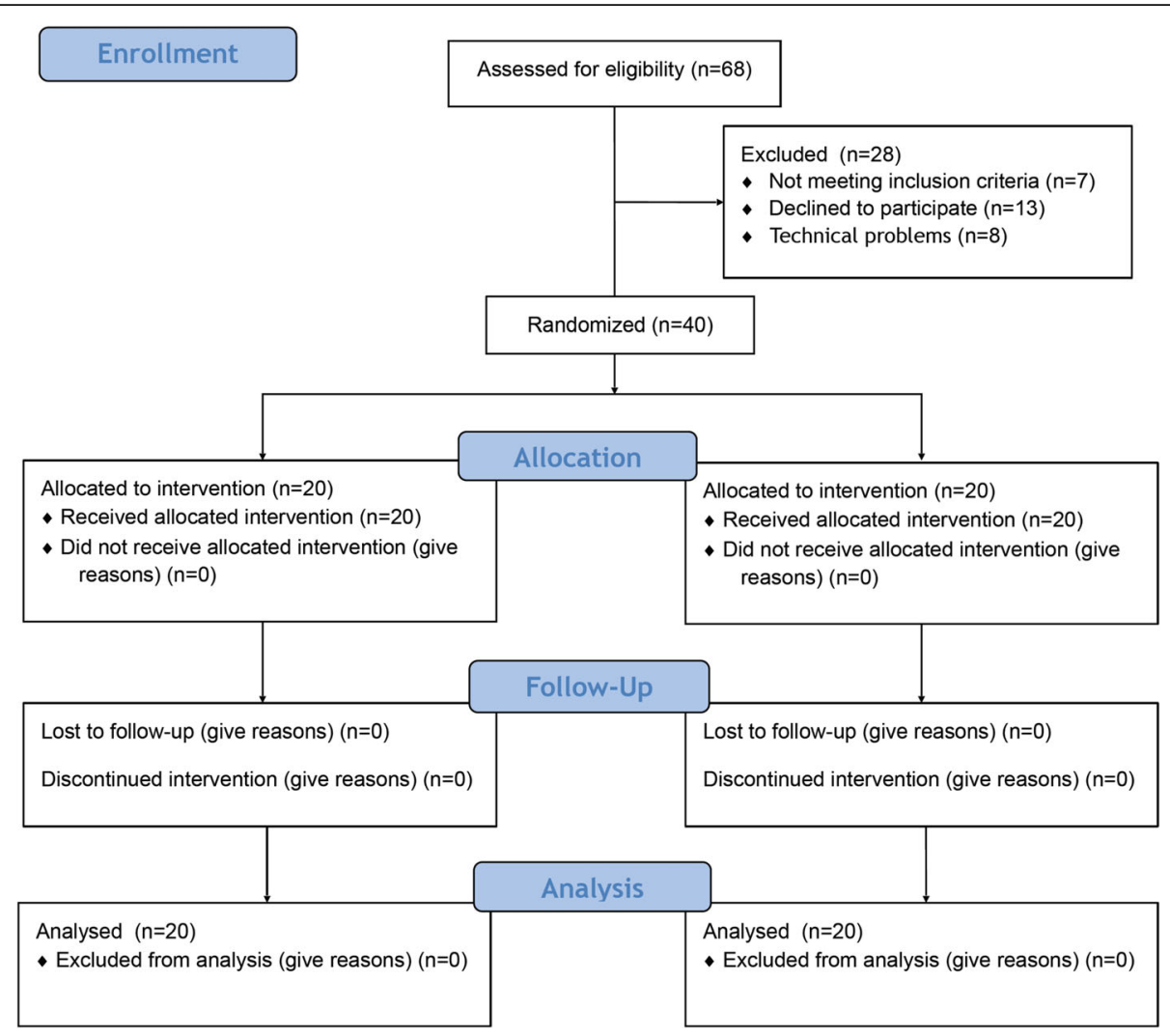

Fig. 2 CONSORT flow diagram. CONsolidated Standards of Reporting Trials (CONSORT) flow diagram showing patients' recruitment and allocation

0.001). There was no interaction between groups and times.

The $\mathrm{StO}_{2}$-ischemic slope decreased after surgery $(P$ value for differences between times $=0.021$; inside each group: T0 vs $\mathrm{T} 1=P<0.02$ ) without any influence of group assignment. The $\mathrm{StO}_{2}$-reperfusion slope was not significantly modified. Baseline brachial artery diameter before occlusion increased after surgery $(P=0.002)$, and was independent of group assignment. Maximal postocclusive dilation (i.e. FMD-max) tended to decrease at T1 and $\mathrm{T} 2$, without reaching statistical significance, because of high interindividual variability. Group assignment did not influence FMD-derived variables. Haemodynamic goals were achieved equally in both groups, and without any difference in cumulative fluid balance intraoperatively or until PACU discharge.

No serious adverse events occurred. Only two patients experienced a minor adverse effect (tinnitus) with lidocaine infusion, immediately after the loading dose. One patient developed a non-life threatening perioperative cardiac arrhythmia, but had been randomized to the PLA group. Mean arterial pressure (MAP) and cardiac index (CI) were significantly lower in the LIDO group; lactates on arrival at PACU were similar between groups (Tables 4 and 5). No other secondary effect occurred during intervention.

\section{Discussion}

In this single-centre, pilot randomized controlled trial we tested the hypothesis that intravenous lidocaine could protect the EG and preserve endothelial function in 40 patients undergoing major abdominal surgery. Anesthesia and the patients' management were strictly controlled and standardized between groups. Patients, healthcare providers and data collectors were blinded to group assignment. In this setting, lidocaine administration failed to show any effect on serum levels of syndecan-1 measured postoperatively or on NIRS- or FMD-derived variables.

Syndecan-1 concentrations increased at most 1.5fold by $24 \mathrm{~h}$ post-surgery in a statistically significant manner $(P<0.001)$, and independently from group assignment. This moderate flaking of EG resembles the results described by Steppan et al. [12] in patients undergoing major abdominal surgery. However, it was much less pronounced than previously described in different clinical scenarios. Syndecan-1 was reported to increase up to 100 -fold in trauma patients [21], 65-fold in patients undergoing major vascular surgery with global and regional ischemia [22], 8-fold in septic patients [12], and 3- to 4-fold after cardiac surgery [23, 24] or after resuscitated cardiac arrest [25]. 
Table 1 Population characteristics. Placebo (PLA) group and lidocaine (LIDO) group, data as mean \pm SD or $n(\%)$

\begin{tabular}{|c|c|c|}
\hline & $\operatorname{PLA}(n=20)$ & $\operatorname{LIDO}(n=20)$ \\
\hline Age (years) & $60 \pm 10.9$ & $63 \pm 12.5$ \\
\hline Male gender & $10(50)$ & $13(65)$ \\
\hline Weight (kg) & $74 \pm 16.5$ & $77 \pm 14.2$ \\
\hline $\mathrm{BMI}(\mathrm{kg} / \mathrm{m} 2)$ & $26 \pm 5.6$ & $26 \pm 4.9$ \\
\hline ASA 1 & $1(5)$ & $1(5)$ \\
\hline ASA 2 & $15(75)$ & $12(60)$ \\
\hline ASA 3 & $4(20)$ & $7(35)$ \\
\hline POSSUM (physiologic) & $16.9 \pm 3.8$ & $19.7 \pm 4.1$ \\
\hline POSSUM (operative) & $12.4 \pm 4.5$ & $11.4 \pm 2.9$ \\
\hline POSSUM predicted morbidity (\%) & $32.4 \pm 23.2$ & $37.2 \pm 18.6$ \\
\hline POSSUM predicted mortality (\%) & $6.4 \pm 9.8$ & $6.8 \pm 6.0$ \\
\hline Cardiac Risk (LEE criteria) (\%) & $1.9 \pm 2.4$ & $1.4 \pm 1.8$ \\
\hline \multicolumn{3}{|l|}{ Comorbidities } \\
\hline Arterial Hypertension & $9(45)$ & $9(45)$ \\
\hline Hypercholesterolemia & $4(20)$ & $3(15)$ \\
\hline Diabetis (typel or II) & $3(15)$ & $5(25)$ \\
\hline Ischemic cardiopathy & $3(15)$ & $2(10)$ \\
\hline Other cardiopathy & 0 & $2(10)$ \\
\hline Tobacco abuse & $6(30)$ & $6(30)$ \\
\hline Renal Insufficiency (KDIGO stage $<3 \mathrm{~b}$ ) & $1(5)$ & $3(15)$ \\
\hline COPD & $3(15)$ & $3(15)$ \\
\hline Stroke or TIA & $1(5)$ & $2(10)$ \\
\hline Peripheral Arterial Disease & $1(5)$ & 0 \\
\hline \multicolumn{3}{|l|}{ Medications } \\
\hline Beta-blockers & $2(10)$ & $7(35)$ \\
\hline Ca-channel blockers & $2(10)$ & 0 \\
\hline ACE - inhibitors & $7(35)$ & $4(20)$ \\
\hline Nitrates & 0 & 0 \\
\hline Oral Antidiabetic Agents & $1(5)$ & $3(15)$ \\
\hline Insuline & $2(10)$ & $2(10)$ \\
\hline Other Medications & $17(85)$ & $11(55)$ \\
\hline
\end{tabular}

Our results should be interpreted in the light of multiple considerations. First, even if the increase in serum levels of syndecan-1 were correlated with mortality [25], it is unknown whether this correlation would be true only beyond a given threshold. We consequently are unable to state if the 1.5-fold increase in syndecan-1 concentrations was clinically relevant. In addition, this focus was not an objective of the trial. Second, it is difficult to directly compare the results obtained from different populations (e.g. trauma, cardiac surgery, sepsis) and different mechanisms of primary insult. Moreover, even when a similar population is taken into account (e.g., major abdominal surgery), it should be emphasised that
Table 2 Surgery performed. Placebo (PLA) group and lidocaine (LIDO) group, data as mean \pm SD or $n(\%)$

\begin{tabular}{lll}
\hline & $\mathrm{PLA}(n=20)$ & $\mathrm{LIDO}(n=20)$ \\
\hline Laparotomy & $11(55)$ & $12(60)$ \\
Laparoscopy & $4(20)$ & $5(25)$ \\
Both & $5(25)$ & $3(15)$ \\
Interventions & & \\
$\quad$ Total Mesorectal Excision & $3(15)$ & $2(10)$ \\
Gastrectomy (total or partial) & $2(10)$ & $3(15)$ \\
Duodeno-Cephalo-Pancreatectomy & $8(40)$ & $4(20)$ \\
Abdominoperineal resection & $1(5)$ & $1(5)$ \\
Surrenalectomy & $1(5)$ & $1(5)$ \\
Ovarian cancer cytoreductive surgery & $2(10)$ & $1(5)$ \\
Extensive retroperitoneal & 0 & $1(5)$ \\
lymphadenectomy & & $6(30)$ \\
Nephrectomy (total or partial) & $2(10)$ & $1(5)$ \\
Cystectomy & $1(5)$ & $228 \pm 80$ \\
Duration, skin-to-skin (min) & $232 \pm 89$ & \\
\hline
\end{tabular}

the study by Steppan et al. [12] lacked detailed information about surgical procedures and the patients' baseline characteristics and perioperative management. Thus, making a direct comparison was not possible. Third, to our knowledge, this is the first randomized controlled trial that studied the effects of a drug on EG in a perioperative setting which had been optimized to reduce endothelial dysfunction. In fact, previous literature reports were primarily observational or focused on a single intervention without any attempt to control the plethora of factors that possibly influence EG disruption (in particular fluids, hemodynamics and corticoid administration) $[8,14,17]$. In our study, fluids and haemodynamic management were goal-directed and patients of both groups received $10 \mathrm{mg}$ dexamethasone. The latter is the standard of care in our institution for postoperative nausea and vomiting prevention and is part of a multimodal strategy for pain control. It is possible that the pronounced and prolonged anti-inflammatory effect of dexamethasone could mask or attenuate any effect of lidocaine. This factor is actually a limitation of this study. However, we decided to maintain this strategy for ethical reasons and in the belief that clinical research should try to improve patients' outcome beyond the best known clinical practice.

Near infrared spectroscopy may be used in combination with a VOT to assess the microcirculatory response to an ischemic challenge, and thus reflect the pre-existing vascular reserve [26]. This technique has been previously used in different clinical populations to assess peripheral microvascular adequacy. We were unable to show any influence of lidocaine on NIRS-derived variables. The $\mathrm{StO}_{2}$-baseline was slightly increased in the 


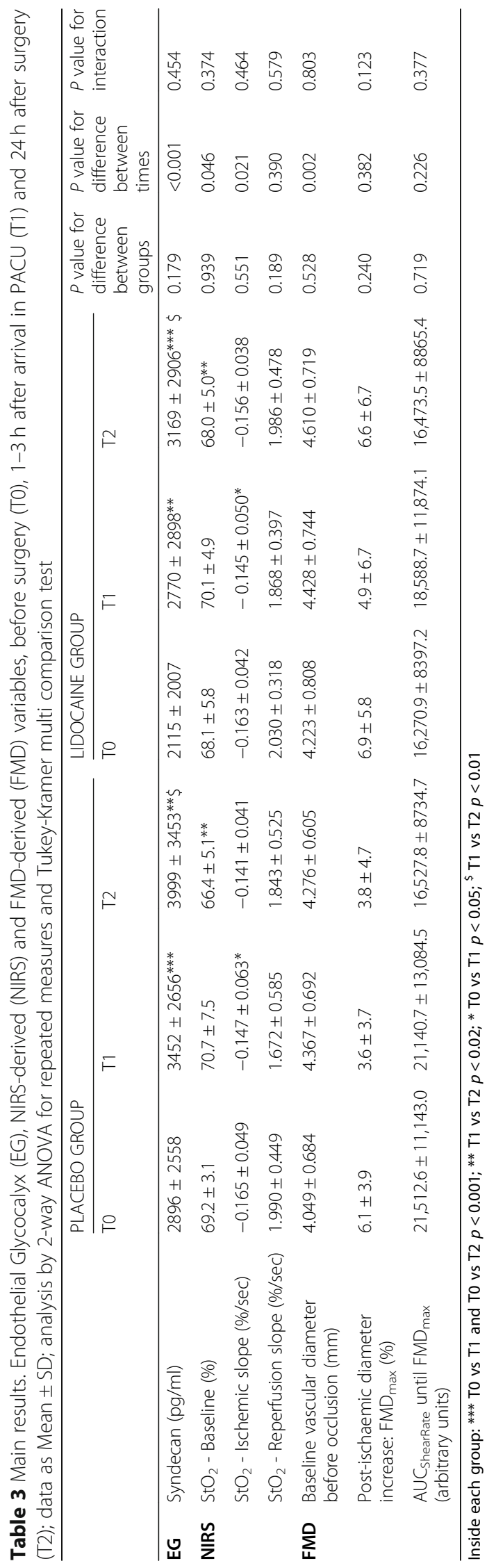


Table 4 Fluid requirements and per-operative haemodymics. Fluid requirements (FLUIDS); peri-operative haemodymics (HD); data as Mean \pm SD - Analysis by Mann-Whitney test

\begin{tabular}{|c|c|c|c|c|}
\hline & & PLACEBO GROUP & LIDOCAINE GROUP & $\begin{array}{l}P \text { value for difference } \\
\text { between groups }\end{array}$ \\
\hline \multirow[t]{5}{*}{ FLUIDS } & Total in during surgery $(\mathrm{ml})$ & $3150 \pm 1822$ & $2410 \pm 948$ & 0.213 \\
\hline & Fluid balance during surgery (ml) & $2237 \pm 1408$ & $1758 \pm 957$ & 0.267 \\
\hline & Total in during PACU stay (ml) & $2820 \pm 1208$ & $2874 \pm 1305$ & 0.968 \\
\hline & Fluid balance during PACU stay (ml) & $1613 \pm 1200$ & $1781 \pm 1204$ & 0.598 \\
\hline & Global fluid balance (ml) & $3850 \pm 1740$ & $3540 \pm 1469$ & 0.607 \\
\hline \multirow[t]{7}{*}{ HD } & $\mathrm{HR}(\mathrm{bpm})$ & $73.2 \pm 11.6$ & $68.7 \pm 10.9$ & 0.307 \\
\hline & MAP (mmHg) & $78.8 \pm 6.8$ & $74.9 \pm 4.1$ & 0.033 \\
\hline & SVI $\left(m L b^{-1} m^{-2}\right)$ & $39.9 \pm 5.1$ & $37.0 \pm 8.8$ & 0.088 \\
\hline & $\mathrm{Cl}\left(\mathrm{L} \mathrm{min}^{-1} \mathrm{~m}^{-2}\right)$ & $2.9 \pm 0.6$ & $2.4 \pm 0.4$ & 0.024 \\
\hline & Percent of time passed with SW < $13(\%)$ & $66.1 \pm 22.7$ & $71.3 \pm 26.0$ & 0.335 \\
\hline & Percent of time passed with MAP $\geq 70 \mathrm{mmHg}(\%)$ & $75.7 \pm 19.0$ & $70.8 \pm 15.4$ & 0.273 \\
\hline & Percent of time passed with $\mathrm{Cl} \geq 2.5 \mathrm{~L} \mathrm{~min}^{-1} \mathrm{~m}^{-2}(\%)$ & $68.8 \pm 35.0$ & $43.6 \pm 32.9$ & 0.025 \\
\hline
\end{tabular}

immediate postoperative period (T1) for both groups, possibly because of receiving oxygen therapy in the recovery room and an increased oxygen delivery to peripheral tissues. The $\mathrm{StO}_{2}$-ischemic slope was significantly different between times but not between groups. The $\mathrm{StO}_{2}$-ischemic slope reflects the balance between oxygen reserves and the metabolic rate of muscle leads under the NIRS sensor [27] (i.e. thenar eminence) and its decrease was possibly because of a decreased metabolic rate of immobile sedated patients in the immediate postoperative period [28]. The $\mathrm{StO}_{2}$-ischemic slope was correlated with $\mathrm{StO}_{2}$-reperfusion slope, owing to the influence of metabolite accumulation on local microvascular reactivity after ischemia [29, 30]; however, it seemed not to be the situation in this cohort. The $\mathrm{StO}_{2}$-reperfusion slope was comparable between groups and showed no relevant modification in the postoperative period. This finding is a strong argument against a substantial alteration of the microvascular reserve.

In the literature, the $\mathrm{StO}_{2}$-reperfusion slope was significantly slower in critically ill patients than in control subjects [31], in septic intensive care unit (ICU) patients than in non-septic ICU patients [32], or in patients undergoing cardiopulmonary bypass (CPB) [33], all conditions that are associated with a substantial microcirculatory impairment. Its decrease in these settings was correlated with a worst outcome [31-33].
With regard to microvascular reactivity, data on endothelial function tested using FMD did not support any effect of lidocaine infusion. The baseline diameter of the brachial artery was significantly increased in the postoperative period, probably because of anesthesia-induced vasodilation. The maximal postischemic dilation of the brachial artery (i.e. FMD-max) was similar in both groups. A trend of FMD-max decrease occurred in the early postoperative period, independently from group assignment. However, it did not reach statistical significance because of high intersubject variability. This result should be interpreted with caution because a significant increase in baseline diameter (approximately 7\%) may result in a decrease in FMD that depends on changes in the resting tone rather than endothelial dysfunction [20]. Moreover, the baseline FMD results suggested that a proportion of patients may have had endothelial dysfunction before surgery, which could attenuate the effects of any intervention in the perioperative setting. Future studies may eventually address this topic further by dividing a population in subgroups, based on baseline analysis of endothelial function. A much more important decrease in FMD-max was recently reported after cardiopulmonary bypass with continuous flow (from 12.8 to $1.6 \%)$ [34], which is a setting associated with increased inflammation, EG disruption and endothelial dysfunction $[23,24]$. Flow-mediated dilation may be intimately

Table 5 Point-of-care laboratory analysis. Point-of-care laboratory analysis (POCT); before surgery (T0); on arrival at PACU (T1); data as Mean \pm SD; analysis by 2-way ANOVA for repeated measures

\begin{tabular}{|c|c|c|c|c|c|c|c|c|}
\hline & & TO & $\mathrm{T1}$ & T0 & $\mathrm{T1}$ & $\begin{array}{l}P \text { value for difference } \\
\text { between groups }\end{array}$ & $\begin{array}{l}P \text { value for difference } \\
\text { between times }\end{array}$ & $\begin{array}{l}P \text { value for } \\
\text { interaction }\end{array}$ \\
\hline \multirow[t]{2}{*}{ POCT } & Lactates (mmol/L) & $0.91 \pm 0.47$ & $1.65 \pm 0.97$ & $1.04 \pm 0.43$ & $1.70 \pm 0.92$ & 0.646 & $<0.001$ & 0.734 \\
\hline & Glycemia (mg/dL) & $122.4 \pm 47.7$ & $164.4 \pm 44.2$ & $126.8 \pm 45.1$ & $191.5 \pm 50.6$ & 0.237 & $<0.001$ & 0.113 \\
\hline
\end{tabular}


linked to EG 'health status', as demonstrated by Yen et al. [35] In their study, flow-induced endothelial nitric oxide production and thus vasodilation were markedly reduced after EG selective enzymatic disruption, which suggests that EG is a key component in transmitting shear stress to endothelial cells. Consistent with the fact that EG was only slightly degraded in our cohort, FMDmax was not expected to show major modifications. Moreover, the $\mathrm{StO}_{2}$-reperfusion slope was recently correlated with FMD-max in healthy volunteers [30]. It was not significantly altered in the study population. Syndecan-1, NIRS and FMD may be complementary means to investigate the same problem: global endothelial dysfunction. Even though we were unable to show a clear correlation between these parameters, to our knowledge this is the first randomized trial to investigate their relationship in the perioperative setting.

There was no significant difference in fluid balance between the two groups. Patients of both groups spent approximately $70 \%$ of surgery time inside the limits imposed by the GDT protocol. Anesthetist reactivity to FloTrac ${ }^{\mathrm{Tm}}$ measurements, time necessary to administer fluids or catecholamines and time the patient needed to respond to the intervention probably accounted for $30 \%$ of time passed outside the protocol limits. Nevertheless, the LIDO group had a significantly lower overall MAP and CI than the placebo group. More patients in the LIDO group needed norepinephrine to maintain haemodynamic goals ( $85 \%$ in the LIDO group vs $60 \%$ in the PLA group), even though the total dose of norepinephrine in patients that actually received it was similar in both groups (data not shown). Consistent with the fact that CI was not a targeted haemodynamic variable, patients in the LIDO group spent only $43.6 \%$ of time with a $\mathrm{CI} \geq 2.5 \mathrm{l} \mathrm{min}^{-1} \mathrm{~m}^{-2}$, compared with patients in the PLA group $(68.8 \%)$. The variability of CI was higher than that of targeted variables (i.e. SVV and MAP). However, postoperative lactate increase on arrival in the PACU was similar in both groups. We did not record any other relevant secondary effect.

This study presents some limits. First, this was a small, single-centre, pilot trial. Our data may not be generalisable, particularly because of differences in surgical management. Second, the use of syndecan-1 as the primary outcome could be controversial because endothelial function is difficult to characterise in the clinical context and it is likely that a single measure is insufficient to describe its complexity. For this reason, we used both NIRS- and FMD-derived variables in an attempt to present a more comprehensive model for the clinical study of endothelial function. However, in our population we failed to demonstrate any major endothelial dysfunction, independent from group assignment and contrary to what was expected based on previous literature findings $[8,9]$. Third, the lack of a positive control (i.e. patients with significant endothelial dysfunction and
EG disruption) is a limit and prevented the ability to demonstrate any significant correlation between syndecan-1 concentrations, $\mathrm{StO}_{2}$-reperfusion slope and FMD-max, as initially hypothesised.

\section{Conclusions}

Based on previous studies demonstrating the antiinflammatory properties of amide-linked LAs [3] and its effects on bowel motility [2], we hypothesised that lidocaine could reduce fluid extravasation and tissue edema by protecting the EG. Even if this was a pilot study, with a reduced population number, endothelial dysfunction in major abdominal surgery is probably overestimated when studied in a controlled setting. Based on our results, the hypothesis that lidocaine could affect endothelial function is unlikely.

\section{Supplementary information}

Supplementary information accompanies this paper at https://doi.org/10. 1186/s12871-020-01075-x.

Additional file 1. Image 1 shows the standard set up for a participant. Additional file 2. Image 2 shows the evolution of $\mathrm{StO}_{2}$ during the test in one participant.

Additional file 3. Image 3 shows the evolution of the brachial artery diameter and shear rate during a test in one participant.

Additional file 4. Consort checklist.

\begin{abstract}
Abbreviations
Cl: Cardiac Index; EG: Endothelial Glycocalyx; FMD: Flow Mediated Dilation; GDT: Goal Directed Therapy; LAs: Locals Anesthetics; LIDO: LIDOcaine Group; MAP: Mean Arterial Pressure; NIRS: Near-Infrared Spectroscopy; PACU: PostAnesthesia Care Unit; PLA: PLAcebo Group; $\mathrm{StO}_{2}$ : Tissue Oxygen Saturation; SW: Systolic Volume Variation; VOT: Vascular Occlusion Test
\end{abstract}

\section{Acknowledgments}

The authors would like to thank the following individuals: Veronique Corbière, Françoise Mascart and Audrey Godefroid of the Laboratory of Vaccinology and Mucosal Immunity, Université Libre de Bruxelles (Brussels, Belgium) for their substantial help in laboratory analysis of syndecan-1; Anita Verleije, Kimberly Gonsette, Shalini Raj-Lawrence and all post-graduate students of the Department of Anaesthesiology of Erasme Hospital for providing care to the study patients and help in data collection; Luc Barvais and Alexandre Joosten for their critical discussion of this study's methods and results.

\section{Presentation}

Société Française d'Anesthésie-Réanimation, 21-23 September 2017 Congress, Paris, France

American Society of Anesthesiologists, 21-25 October 2017 Meeting, Boston, MA, USA

\section{Authors' contributions}

All authors have read and approved the manuscript. All authors contributed to the interpretation of results and revision of manuscript, as follows: M.P. and L.V.O.: study concept and design; M.P.: patient recruitment, measurements and data collection, laboratory analysis and redaction of the manuscript draft; E.E.: randomization and statistical analysis; and N.G., K.T. and B.I.: intra-operative management of participants. M.P. presented partial study data as an e-communication at the 2017 Société Française d'Anesthésie-Réanimation annual congress held in Paris, France between 21 and 23

September 2017. B.I. presented the study as e-communication at the 2017 
American Society of Anesthesiologists meeting held in Boston, MA, USA between 21 and 25 October 2017

\section{Funding}

This study was supported by a scholarship for clinical research endorsed by the Medical Council of Erasme Hospital (Brussels, Belgium) but the institution did not analyze the datas nor the manuscript and did not have any influence on the study design.

\section{Availability of data and materials}

The datasets generated and/or analysed during the current study are not publicly available due technical reasons, but are available from the corresponding author on reasonable request.

\section{Ethics approval and consent to participate}

Ethical approval for this study (Ethics Committee No. P2016/404/2016003918-27) was provided by the Ethics Committee Erasme Hospital, 808 route de Lennik, B-1070 Brussels, Belgium (Chairperson Prof J.-M. Boeynaems) on 24 October 2016. All included patients signed a written informed consent before participation.

\section{Consent for publication}

$$
\text { Not applicable. }
$$

\section{Competing interests}

The authors declare that they have no competing interests.

\section{Author details}

${ }^{1}$ Department of Anesthesiology, Centre Hospitalier Universitaire Grenoble-Alpes, Boulevard de la Chantourne, 38700 Grenoble, France. ${ }^{2}$ Department of Anesthesiology, Erasme Hospital, Université Libre de Bruxelles, Brussels, Belgium. ${ }^{3}$ Department of Anesthesiology, CHIREC Hospital group, Brussels, Belgium.

\section{Received: 13 March 2020 Accepted: 16 June 2020}

\section{Published online: 23 June 2020}

\section{References}

1. Hollmann MW, Durieux ME. Local anesthetics and the inflammatory response: a new therapeutic indication? Anesthesiology. 2000;93:858-75.

2. Blumenthal $S$, Borgeat $A$, Pasch $T$, et al. Ropivacaine decreases inflammation in experimental endotoxin-induced lung injury. Anesthesiology. 2006;104: 961-9.

3. Allegri M, Bugada D, De Gregori $M$, et al. Continuous wound infusion with chloroprocaine in a pig model of surgical lesion: drug absorption and effects on inflammatory response. J Pain Res. 2017;10:2515-24.

4. Saito T, Yamamoto $Y$, Feng GG, Kazaoka Y, Fujiwara $Y$, Kinoshita H. Lidocaine prevents oxidative stress-induced endothelial dysfunction of the systemic artery in rats with intermittent periodontal inflammation. Anesth Analg. 2017;124:2054-62

5. Piegeler T, Votta-Velis EG, Bakhshi FR, et al. Endothelial barrier protection by local anesthetics: ropivacaine and lidocaine block tumor necrosis factor-ainduced endothelial cell Src activation. Anesthesiology. 2014;120:1414-28.

6. Kranke $\mathrm{P}$, Jokinen J, Pace $\mathrm{NL}$, et al. Continuous intravenous perioperative lidocaine infusion for postoperative pain and recovery. Cochrane Database Syst Rev. 2015;7:CD009642 Kranke P (editor): Chichester: Wiley.

7. Becker BF, Chappell D, Bruegger D, Annecke T, Jacob M. Therapeutic strategies targeting the endothelial glycocalyx: acute deficits, but great potential. Cardiovasc Res. 2010:87:300-10.

8. Woodcock TE, Woodcock TM. Revised Starling equation and the glycocalyx model of transvascular fluid exchange: an improved paradigm for prescribing intravenous fluid therapy. Br J Anaesth. 2012;108:384-94.

9. Jung C, Fuernau G, Muench $P$, et al. Impairment of the endothelial glycocalyx in cardiogenic shock and its prognostic relevance. Shock. 2015; 43:450-5.

10. De Backer D, Donadello K, Taccone FS, Ospina-Tascon G, Salgado D, Vincent J. Microcirculatory alterations: potential mechanisms and implications for therapy. Ann Intensive Care. 2011:1:27.

11. Creteur J, Carollo T, Soldati G, Buchele G, De Backer D, Vincent JL. The prognostic value of muscle StO 2 in septic patients. Intensive Care Med. 2007;33:1549-56.
12. Steppan J, Hofer S, Funke B, et al. Sepsis and major abdominal surgery lead to flaking of the endothelial glycocalix. J Surg Res. 2011;165:136-41.

13. Ekeloef S, Larsen MH, Schou-Pedersen AM, Lykkesfeldt J, Rosenberg J, Gögenür I. Endothelial dysfunction in the early postoperative period after major colon cancer surgery. Br J Anaesth. 2017;118:200-6.

14. Guidet B, Ait-Oufella H. Fluid resuscitation should respect the endothelial glycocalyx layer. Crit Care. 2014;18:707.

15. Chappell D, Jacob M, Hofmann-Kiefer K, et al. Hydrocortisone preserves the vascular barrier by protecting the endothelial glycocalyx. Anesthesiology. 2007;107:776-84.

16. Chappell D, Heindl B, Jacob M, et al. Sevoflurane reduces leukocyte and platelet adhesion after ischaemia-reperfusion by protecting the endothelial glycocalyx. Anaesthesiology. 2011;115:483-91.

17. Bashandy GM. Implications of recent accumulating knowledge about endothelial glycocalyx on anesthetic management. J Anesth. 2015;29:269-78.

18. Wodack K, Pope A, Tomköetter L, et al. Individualized early goal-directed therapy in systemic inflammation. Crit Care Med. 2014;42:e741-51.

19. Gemignani V, Bianchini $E$, Faita F, et al. Ultrasound measurement of the brachial artery flow-mediated dilation without ECG gating. Ultrasound Med Biol. 2008:34:385-91.

20. Corretti MC, Anderson TJ, Benjamin EJ, et al. Guidelines for the ultrasound assessment of endothelial-dependent flow-mediated vasodilation of the brachial artery: a report of the international brachial artery reactivity task force. J Am Coll Cardiol. 2002:39:257-65.

21. Rahbar E, Cardenas JC, Baimukanova G, et al. Endothelial glycocalyx shedding and vascular permeability in severely injured trauma patients. J Transl Med. 2015;13:17.

22. Rehm M, Bruegger $D$, Christ $F$, et al. Shedding of the endothelial glycocalyx in patients undergoing major vascular surgery with global and regional ischaemia. Circulation. 2007;116:1896-906.

23. Svennevig K, Hoel T, Thiara A, et al. Syndecan-1 plasma levels during coronary artery bypass surgery with and without cardiopulmonary bypass. Perfusion. 2008:23:165-71.

24. Bruegger D, Brettner F, Rossberg I, et al. Acute degradation of the endothelial glycocalyx in infants undergoing cardiac surgical procedures. Ann Thorac Surg. 2015;99:926-31.

25. Grundmann S, Fink K, Rabadzhieva L, et al. Perturbation of the endothelia glycocalyx in post cardiac arrest syndrome. Resuscitation. 2012;83:715-20.

26. Gomez H, Torres A, Polanco P, Zenker S, Pinsky MR. Use of non-invasive NIRS during a vascular occlusion test to assess dynamic tissue $\mathrm{O} 2$ saturation response. Intensive Care Med. 2008;34:1600-7.

27. Creteur J. Muscle StO2 in critically ill patients. Curr Opin Crit Care. 2008;14: 361-6.

28. Mesquida J, Gruartmoner G, Espinal C. Skeletal muscle oxygen saturation (StO2) measured by near-infrared spectroscopy in the critically ill patients. Biomed Res Int. 2013:2013:502194.

29. Mayeur C, Campard S, Richard C, Teboul JL. Comparison of four different vascular occlusion tests for assessing reactive hyperemia using near-infrared spectroscopy. Crit Care Med. 2011;39:695-701.

30. McLay KM, Fontana FY, Nederveen JP, et al. Vascular responsiveness determined by near-infrared spectroscopy measures of oxygen saturation. Exp Physiol. 2016;101:34-40.

31. Orbegozo Cortés $D$, Rahmania L, Irazabal M, et al. Microvascular reactivity is altered early in patients with acute respiratory distress syndrome. Respir Res. 2016:17:59.

32. Creteur J, Carollo T, Soldati G, Buchele G, De Backer D, Vincent JL. The prognostic value of muscle $\mathrm{StO} 2$ in septic patients. Intensive Care Med. 2007;33:1549-56.

33. Monthé-Sagan K, Fischer MO, Saplacan V Gerard JL, Hanouz JL, Fellahi JL. Near-infrared spectroscopy to assess microvascular dysfunction: a prospective pilot study in cardiac surgery patients. J Crit Care. 2016:31:264-8.

34. Sangalli F, Guazzi M, Senni S, et al. Assessing endothelial responsiveness after cardiopulmonary bypass: insights on different perfusion modalities. J Cardiothorac Vasc Anesth. 2015;29:912-6.

35. Yen W, Cai B, Yang J, et al. Endothelial surface glycocalyx can regulate flowinduced nitric oxide production in microvessels in vivo. PLoS One. 2015;10: e0117133.

\section{Publisher's Note}

Springer Nature remains neutral with regard to jurisdictional claims in published maps and institutional affiliations. 